\title{
Pressure Analysis in the Draft Tube of a Pump-Turbine under Steady and Transient Conditions
}

\author{
Jing Yang ${ }^{1}$, Yue Lv ${ }^{2}$, Dianhai Liu ${ }^{1}$ and Zhengwei Wang ${ }^{3, *}$ \\ 1 Pumped-Storage Technological \& Economic Research Institute, State Grid Xin Yuan Company Ltd., \\ Beijing 100761, China; yangjingshirley@163.com (J.Y.); dianhai-liu@sgxy.sgcc.com.cn (D.L.) \\ 2 College of Water Resources and Civil Engineering, China Agricultural University, Beijing 100083, China; \\ sdzbly1015@163.com \\ 3 Department of Energy and Power Engineering, Tsinghua University, Beijing 100084, China \\ * Correspondence: wzw@mail.tsinghua.edu.cn; Tel.: +86-010-62791262
}

Citation: Yang, J.; Lv, Y.; Liu, D.; Wang, Z. Pressure Analysis in the Draft Tube of a Pump-Turbine under Steady and Transient Conditions. Energies 2021, 14, 4732. https:// doi.org/10.3390/en14164732

Academic Editor: Marco Torresi

Received: 18 June 2021

Accepted: 19 July 2021

Published: 4 August 2021

Publisher's Note: MDPI stays neutral with regard to jurisdictional claims in published maps and institutional affiliations.

Copyright: (c) 2021 by the authors. Licensee MDPI, Basel, Switzerland. This article is an open access article distributed under the terms and conditions of the Creative Commons Attribution (CC BY) license (https:/ / creativecommons.org/licenses/by/ $4.0 /)$.

\begin{abstract}
Pumped-storage power stations play a regulatory role in the power grid through frequent transition processes. The pressure pulsation in the draft tube of the pump-turbine under transient processes is important for safe operation, which is more intense than that in the steady-state condition. However, there is no effective method to obtain the exact pressure in the draft tube in the transient flow field. In this paper, the pressure in the draft tube of a pump-turbine under steady-state and transient conditions are studied by means of CFD. The reliability of the simulation method is verified by comparing the real pressure pulsation data with the test results. Due to the distribution of the pressure pulsation in the draft tube being complex and uneven, the location of the pressure monitoring points directly affects the accurate judgement of cavitation. Eight monitoring surfaces were set in the straight cone of the draft tube and nine monitoring points were set on each monitoring surface to analyze the pressure differences on the wall and inside the center of the draft tube. The relationships between the pressure pulsation value inside the center of the draft tube and on the wall are studied. The "critical" wall pressure pulsation value when cavitation occurs is obtained. This study provides references for judging cavitation occurrences by using the wall pressure pulsation value in practical engineering.
\end{abstract}

Keywords: pump turbine; draft tube; pressure pulsation; transient process; cavitation

\section{Introduction}

Different from conventional power and pumping stations, a pumped-storage power station has a long water conveyance system, which means the transition process's effect on the flow in the pump-turbine is more significant. The field test results show that there is a significant amount of pressure pulsations at the draft tube during the transition process [1,2]. Some power stations even suffer from structural damage due to severe unit vibration [3].

Luo X. et al. [4] calculated and analyzed the runaway transient process of a tubular turbine, and found that a strong pressure pulsation was generated in the draft tube, at up to $104 \%$ of the test head, which threatened the safe and stable operation of the unit. Liu J. et al. [5] simulated the flow of a Francis turbine under runaway conditions and found that the pressure pulsation in the channel was as high as 16\%. Furthermore, the characteristic curve changes significantly in the case of load rejections under different heads based on the characteristic curve of the pump-turbine. Thus, the pulsations in the flow field must be different. All these studies confirm that the pressure pulsation in a transient flow field is significantly stronger than that in a steady flow field, and the study of pressure pulsation in transient processes is an important aspect to ensure the safe and stable operation of the unit. However, it is difficult to measure the pulsation in a field unit operation, and 
more effective methods are needed to study the pulsation in the extreme conditions of the transition process.

Three dimensional CFD calculation is an effective method to obtain the pressure pulsation in the channel. At present, it is widely used in steady-state calculation and three-dimensional transient flow research of conventional water turbines [6], such as a pump [7], as well as in tubular, axial-flow, and Francis turbines with a short water delivery channel. However, when the water conveyance system is long, considering the three-dimensional calculation of the whole system, especially the cavitation model, the calculation will consume a lot of resources. In order to obtain the transient flow characteristics of the pipeline and the unit at the same time, the one-dimensional and three-dimensional coupling method is more reasonable. In this method, three-dimensional CFD is used for the pump-turbine, and one-dimensional CFD is used for other parts of the pipe system. The interface is set for real-time data exchange to ensure that the boundary conditions of the three-dimensional and one-dimensional models can be updated to advance the calculation.

Ruprecht [8] also realized the influence of draft tube flow on the hydraulic system under part load by using one-dimensional and partial three-dimensional simulation methods. You J. et al. [9] studied the cavitation characteristics of the pump-turbine and the pressure pulsation characteristics of the pump's powered-off runaway transition process by using the three-dimensional CFD method and one-dimensional three-dimensional coupling method, respectively. Li Z. [10] and Zhang X. et al. [11] used this method to simulate the runaway transition process of a pump-turbine, and the results showed that the pressure pulsation obtained by this method is in good agreement with the model test. Cherny $\mathrm{S}$. et al. [12] also studied the transient flow of a hydraulic turbine by the coupling method. In the three-dimensional calculation, the reflection and superposition of the water hammer pressure in the one-dimensional pipeline were considered in the coupled calculation model. Therefore, in the process of guide vane closing, the calculated inlet pressure of the volute will rise higher, and the pressure pulsation in three-dimensional unit flow field is more severe, which can more truly reflect the interaction between the pipeline transient flow and three-dimensional transient flow in the unit.

Because the transition process will pass through a variety of operation areas in a short time, the actual process is often accompanied by the rise and fall of the pressure in the flow channel. The low pressure in the draft tube may cause a short-term cavitation phenomenon, which will significantly change the wave velocity of the local flow field and affect the stability of the whole hydraulic system of the hydropower station. For example, when Yang J. [13] conducted numerical studies on cavitation in a draft tube, it was found that the wave velocity in the cone tube may also be reduced to about $50 \mathrm{~m} / \mathrm{s}$ when the volume of the vortex band accounts for a large proportion. However, we usually choose a fixed value of $1200 \mathrm{~m} / \mathrm{s}$ in the calculation of the one-dimensional transition process, which is far from the same. Nicolet [14,15] and Alligné [16] et al. predicted the abnormal pressure vibration and surge phenomenon of power stations considering the influence of draft tube cavitation on the one-dimensional transient process. Meng L. [17] explored the influence of cavitation on the start-up process of a centrifugal pump by coupling the one-dimensional calculation software Flowmaster with the three-dimensional calculation software Fluent. The results show that the transient characteristics of the cavitation are more complicated than the results of an ordinary steady-state cavitation calculation when cavitation occurs in the transient process, because the transient process will pass through many operating regions in a short time.

In addition, the dynamic characteristics and collapse of the cavitation chamber may cause extreme accidents, such as "lift", which has been confirmed by many researchers. For example, Zhang X. [18] et al. have studied the cavitation of a draft tube during the transition process. They think that the larger the volume of the cavitation chamber in the draft tube, the more significant the sudden increase in pressure caused by collapse, and the greater the additional impact on the runner in the axial and radial directions. Pejovic $S$. 
et al. [19] pointed out that in the reverse-S region of the pump-turbine, the phenomenon of liquid column separation and bridging may occur due to cavitation, and there will be a large counter water pressure. The phenomenon of pump-turbine backwater hammer shows that when the suction height of the pump-turbine is large, local cavitation will appear at the bottom of the discharge cone, and the cavitation chamber will expand and collapse, which will form a large backwater hammer pressure.

It can be seen that the cavitation flow field in the draft tube affects the large pulsation results of the flow field of the pump-turbine. The influence of cavitation on stability should also be considered in the analysis of the transition process. At present, few studies focus on the cavitation characteristics of the transient process, with the research on draft tube cavitation mainly aimed at the modeled runner calculation and experimented with in a steady flow field [20].

In engineering, the draft tube pressure pulsation is often monitored by setting a pressure transducer on the wall to judge whether cavitation occurs in a pump-turbine and hydro-turbine. However, due to the existence of a vortex, the pressures on the wall and inside the center of the draft tube are different. Furthermore, according to the results of steady cavitation research, the cavitation vortex rope appears first in the central region of the draft tube. The pressure inside the center of the draft tube is a more accurate reference to judge cavitation, which is impossible to get.

Therefore, in order to accurately reflect the internal flow characteristics of the unit in the transient process, it is necessary to further study the pressure pulsation in the transient process of a pumped-storage power station through one-dimensional and threedimensional coupling methods, and analyze the pressure differences in the central region and wall of the draft tube under the transient process. This also forms the basis for improving the runner optimization design and putting forward reasonable control measures to ensure the safe and stable operation of the power plant.

\section{Research Object and Numerical Calculation Method}

\subsection{Research Object}

In this paper, a pump-turbine is taken as the research object. The calculation domains, including the spiral casing, guide vane, stay vane, runner, and draft tube were modeled, as shown in Figure 1a. The computing domain of the one-dimensional calculation is shown in Figure 1b, which includes an upstream reservoir, downstream reservoir, gate shaft, diversion tunnel, high-pressure pipeline, pump-turbine, and tailrace tunnel.

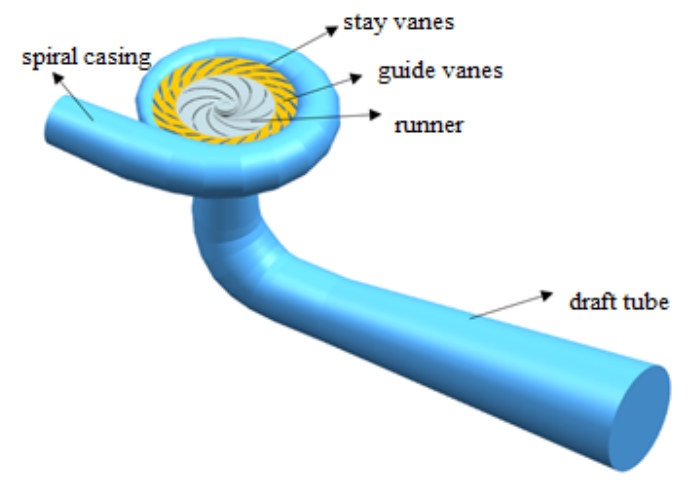

(a) Three-dimensional computational fluid domain

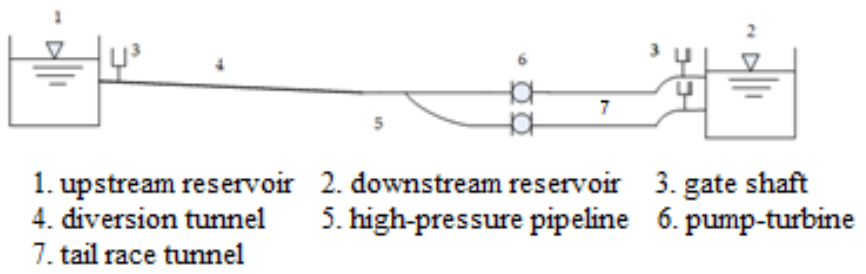

(b) One-dimensional computational domain

Figure 1. Computational model (3D and 1D).

The rated speed of the pump-turbine is $300 \mathrm{rpm}$, the specific speed is 167.3 (specific speed $n_{s}=3.65 n \sqrt{q_{V}} / H^{3 / 4}$ ), the number of guide vanes and stay vanes is both 20 , and the number of runner blades is 9 . The runner diameter is $5.4 \mathrm{~m}$. The diameter of the spiral casing inlet is $2.8 \mathrm{~m}$ and the draft tube inlet is $2.7 \mathrm{~m}$. The normal water level of the upper reservoir is $400 \mathrm{~m}$, and the minimum water level is $376.5 \mathrm{~m}$; the normal water level of 
the lower reservoir is $103.7 \mathrm{~m}$, and the minimum water level is $65 \mathrm{~m}$. The length of the diversion pipeline is about $1078 \mathrm{~m}$, and that of the tailrace pipeline is about $472 \mathrm{~m}$.

\subsection{Numerical Calculation Method}

In the three-dimensional simulation, the quality of the grid has a great influence on the calculation results. Considering the calculation resources, calculation accuracy, and grid quality, after the grid independence test, the number of total cells used was 6,823,783. The mesh statistics are shown in Table 1. In this study, the grid density for the runner and draft tube are set higher to capture a more detailed flow for further analyzing.

Table 1. Detailed statistics of the mesh.

\begin{tabular}{|c|c|c|c|c|c|}
\hline Component & $\begin{array}{l}\text { Spiral Casing } \\
+ \text { Stay Vanes }\end{array}$ & Guide Vanes & Runner & Draft Tube & Total \\
\hline Number & $1,980,196$ & 689,676 & $2,451,015$ & $3,922,926$ & \\
\hline Proportion & $29.02 \%$ & $10.11 \%$ & $35.92 \%$ & $57.49 \%$ & $6,823,783$ \\
\hline Type & Unstructured & unstructured & structured & structured & \\
\hline
\end{tabular}

The corresponding measuring points were arranged in 8 sections of the draft tube, as shown in Figure 2a. A total of 9 measuring points were set at each section, as shown in Figure $2 \mathrm{~b}$. The monitoring point marked as P1-1 indicates the number of the monitoring point P1 on Plane 1. The specific parameters of the section position are shown in Table 2, where $\mathrm{z}$ is the $\mathrm{Z}$-axis position of the monitoring section, the reference value is the center line of the guide vanes, and $\mathrm{r}$ is the radius of the plane.

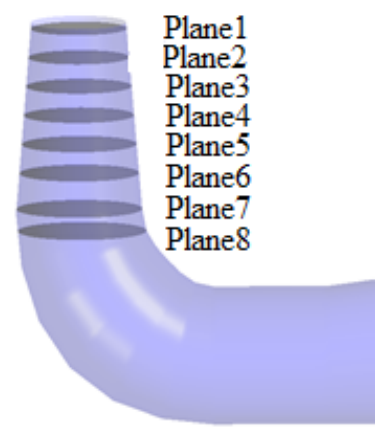

(a) Layout of monitoring surface

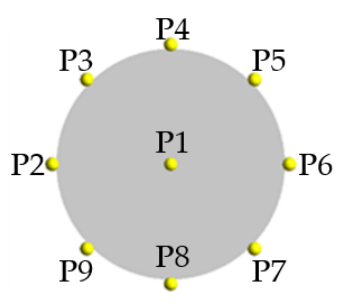

(b) Layout of monitoring points

Figure 2. Distribution of the measuring points in the draft tube.

Table 2. Detailed statistics of the monitoring section.

\begin{tabular}{ccc}
\hline Monitoring Section & $\mathbf{z} / \mathbf{m}$ & $\mathbf{r} / \mathbf{m}$ \\
\hline Plane 1 & -1.907 & 1.410 \\
Plane 2 & -2.667 & 1.47 \\
Plane 3 & -3.427 & 1.53 \\
Plane 4 & -4.187 & 1.59 \\
Plane 5 & -4.947 & 1.65 \\
Plane 6 & -5.707 & 1.71 \\
Plane 7 & -6.647 & 1.77 \\
Plane 8 & -7.227 & 1.82 \\
\hline
\end{tabular}

In this paper, three-dimensional calculation is the main method for the analysis of the steady-state flow. The one-dimensional and three-dimensional coupling method was adopted for the study of the transient condition. Considering the calculation resources and calculation time, an indirect coupling method was adopted in this study. A onedimensional numerical method was used to simulate the load rejection transient process, 
and the results were compared with the test results. According to the one-dimensional calculation results, the pressure values at the spiral casing inlet and draft tube outlet used in the three-dimensional simulations were obtained. The pressure value was calculated according to the water level upstream and downstream of the reservoir and the pipeline loss in the one-dimensional calculation.

Sliding mesh was used at the interface of the guide vane and runner and runner and draft tube. The SST $k$ - $\omega$ model was selected as the turbulence model. The time step of the unsteady calculation under the steady-state condition was a 1/200 rotation period, $\triangle t=0.001 \mathrm{~s}$, and the maximum number of iterations at each time step was set to 20 , and the convergence residual set as $1 \times 10^{-4}$.

For the calculation of cavitation multiphase flow in the hydraulic machinery, the homogeneous model is generally used, which assumes that all of the phases share the turbulent field, using a cavitation model to describe the mass transfer rate between the phases simulated. In this study, the ZGB (Zwart-Gerber-Belamri) cavitation model [21] was selected as the cavitation model, and the saturated vapor pressure was set at $3540 \mathrm{~Pa}$.

The ZGB cavitation model uses the following evaporation and condensation terms:

$$
\begin{gathered}
\dot{m}_{\mathrm{e}}=F_{\mathrm{vap}} \frac{3 \alpha_{\mathrm{nuc}}\left(1-\alpha_{\mathrm{v}}\right) \rho_{\mathrm{v}}}{R_{\mathrm{B}}} \sqrt{\frac{2}{3} \frac{p_{\mathrm{v}}-p}{\rho_{\mathrm{l}}}}, \text { when } p<p_{\mathrm{v}} \\
\dot{m}_{\mathrm{c}}=F_{\text {cond }} \frac{3 \alpha_{\mathrm{v}} \rho_{\mathrm{v}}}{R_{\mathrm{B}}} \sqrt{\frac{2}{3} \frac{p-p_{\mathrm{v}}}{\rho_{\mathrm{l}}}}, \text { when } p \geq p_{\mathrm{v}}
\end{gathered}
$$

The bubble radius $R_{\mathrm{B}}$ is replaced by the gas core radius $R_{\text {nuc }}$, which represents the volume fraction of the gas core; $F_{\mathrm{vap}}$ and $F_{\text {cond }}$ are the evaporation and condensation coefficient, respectively. The default values of each parameter are $R_{\text {nuc }}=1 \times 10^{-6} \mathrm{~m}$, $\alpha_{\text {nuc }}=5 \times 10^{-4}, F_{\text {vap }}=50$, and $F_{\text {cond }}=0.01$.

\section{Analysis of the Results of the Steady-State Conditions}

Firstly, the steady-state condition of a $100 \%$ load was numerically simulated, and the upstream and downstream reservoir water level was 388.7 and 92.23 , respectively. The calculated guide vane opening of $31.63^{\circ}$ was given according to the real machine test data.

In order to verify the pressure pulsation calculation results in this paper, the pressure monitoring points at the real machine were set at the corresponding locations during simulation, which are shown in Figure 3.

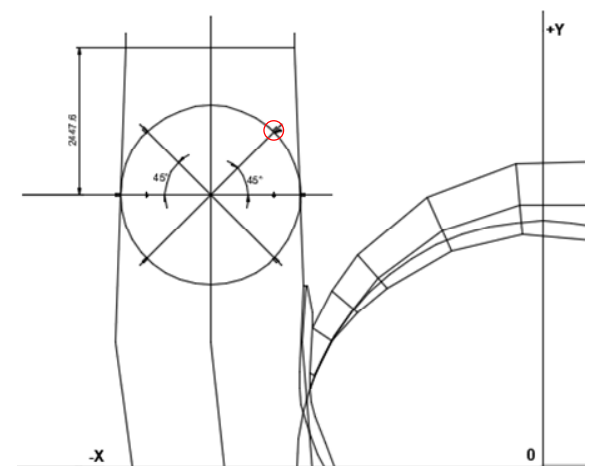

(a) Layout of the pressure pulsation measuring points at the volute inlet

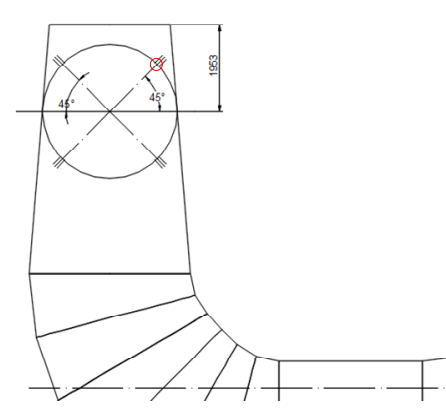

(b) Layout of the pressure pulsation measuring points at the draft tube inlet

Figure 3. Diagram of the pressure pulsation measuring point location.

Due to the difference in time step between the experimental value $(0.01 \mathrm{~s})$ and the calculated value $(0.001 \mathrm{~s})$, it is impossible to make a good comparison and fitting in the frequency domain. So, the mean time value in a rotation cycle was compared, and the 
results are shown in Table 3. It can be seen that the mean pressure at the spiral casing inlet is in good agreement with the calculated value, and the mean pressure pulsation at the draft tube inlet has an error of about $8 \%$. The difference is related to the wall roughness of the model and the inaccurate estimation of pipeline loss.

Table 3. Comparison of the mean values under pressure.

\begin{tabular}{ccc}
\hline & $\mathbf{1 0 0} \%$ Loading & \\
\hline \multirow{2}{*}{ Volute inlet pressure (MPa) } & Experimental value & 3.51 \\
& Simulation value & 3.50 \\
\hline \multirow{2}{*}{ Draft tube inlet pressure $(\mathrm{MPa})$} & Experimental value & 0.78 \\
& Simulation value & 0.845 \\
\hline
\end{tabular}

\subsection{Steady-State Condition without Cavitation}

The pressure distribution in the draft tube in a rotation cycle under $100 \%$ load condition is shown in Figure 4, in which $T$ is the rotation period of the runner. It can be seen that there is an obvious low-pressure area at the cone of the draft tube, and the pressure difference at the wall and at the center of draft tube is large. We also analyzed the pressure at Plane 4, which is shown in Figure 5. It can be seen that there is a low-pressure area in the center of the straight cone section of draft tube. On the same cross section, the pressure difference at the different measuring points is about $1 \times 10^{5} \mathrm{~Pa}$. Thus, it is necessary to study the pressure pulsation at different locations.

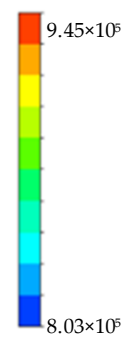

$\mathrm{Pa}$

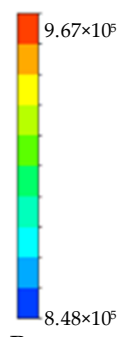

$\mathrm{Pa}$

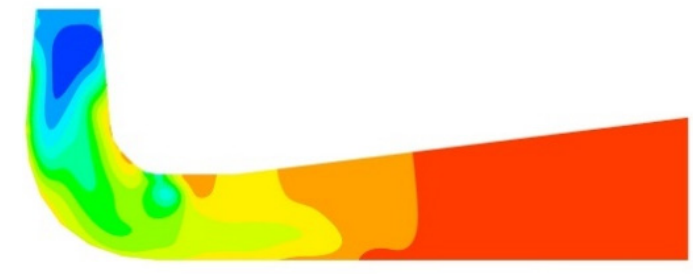

(a) $0 \mathrm{~T}$

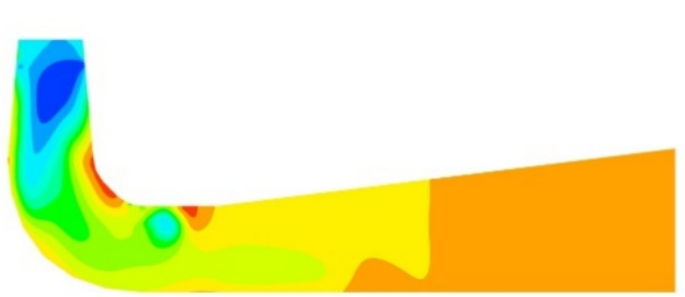

(c) $0.5 T$
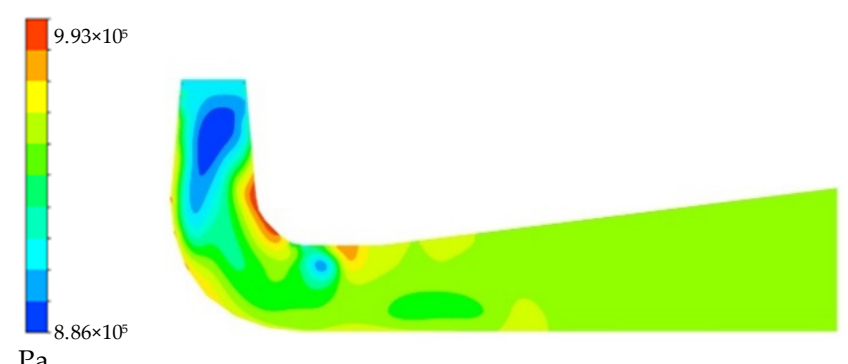

(b) $0.25 T$

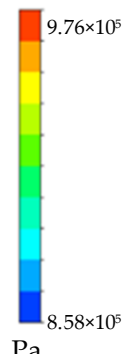

$\mathrm{Pa}$

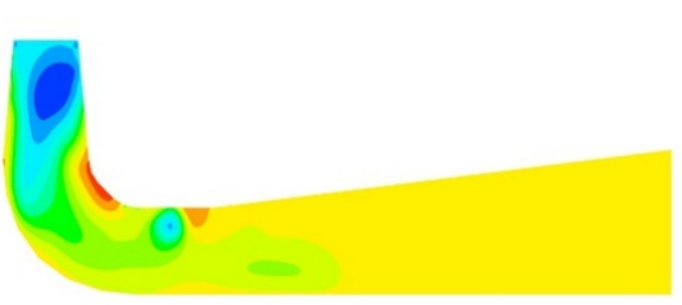

(d) $0.75 \mathrm{~T}$

Figure 4. Pressure distribution in the middle section of draft tube. 


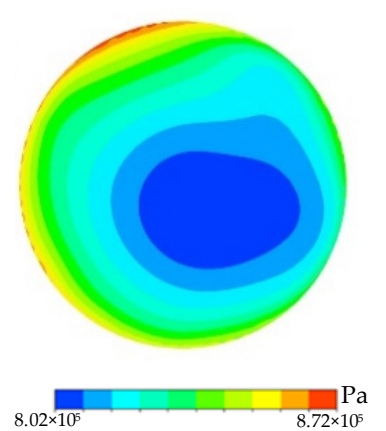

(a) $0 \mathrm{~T}$

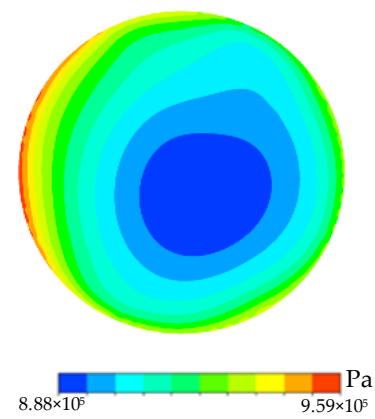

(b) $0.25 T$

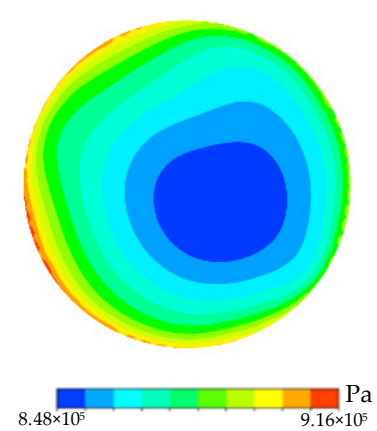

(c) $0.5 \mathrm{~T}$

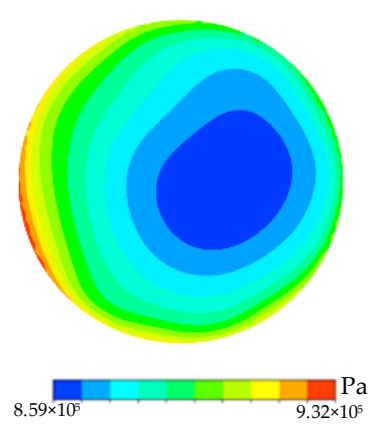

(d) $0.75 \mathrm{~T}$

Figure 5. Pressure distribution of draft tube of Plane 4.

\subsection{Steady-State Condition of Cavitation}

In order to ensure the operation safety of the unit, the pumped-storage power station is usually designed with no cavitation operation. Therefore, it is impossible to simulate cavitation under the given upstream and downstream water level according to field test results. In order to study the pressure pulsation in the cavitation flow field, the pressure value at the outlet boundary conditions was reduced according to the Thoma number shown in (3), to simulate the cavitation flow. Then, the pressure pulsation of the measuring points when serious cavitation occurs in draft tube and the pressure values, obtained from the field test, were analyzed.

$$
\sigma=\left(\frac{P_{\text {out }}}{\rho \mathrm{g}}-h_{z}-\frac{P_{v}}{\rho \mathrm{g}}\right) / H
$$

where $P_{\text {out }}$ is the outlet pressure in draft tube; $P_{v}$, the saturated vapor pressure ( $3540 \mathrm{~Pa}$ ); $h_{z}$, the suction height benchmarked against the center of guide vane; and $H$, the net head.

To compare the pressure near the wall and at the center point in the draft tube for the forecast of cavitation, the pressure pulsation of each monitoring point was processed. Firstly, the mean pressure of each monitoring point P2-P9 on a monitoring surface within the whole period ( 5 rotation cycles were taken in this paper) was calculated. Then, the mean pressure of P2-P9 in a section was averaged again. Finally, the averaged wall pressure and the center point pressure on each section without cavitation and with cavitation are shown in Figure 6. It can be seen that the central monitoring point obviously is the most accurate point to judge cavitation, no matter the cavitation being present or not. Furthermore, the pressure distribution in the draft tube is quite different with or without cavitation. When severe cavitation occurs in the draft tube, the pressure value of the center point is always the saturated vapor pressure of $3540 \mathrm{~Pa}$. However, the average pressure on the wall is quite different. It is necessary to find a reasonable way to judge the pressure in the draft tube. 


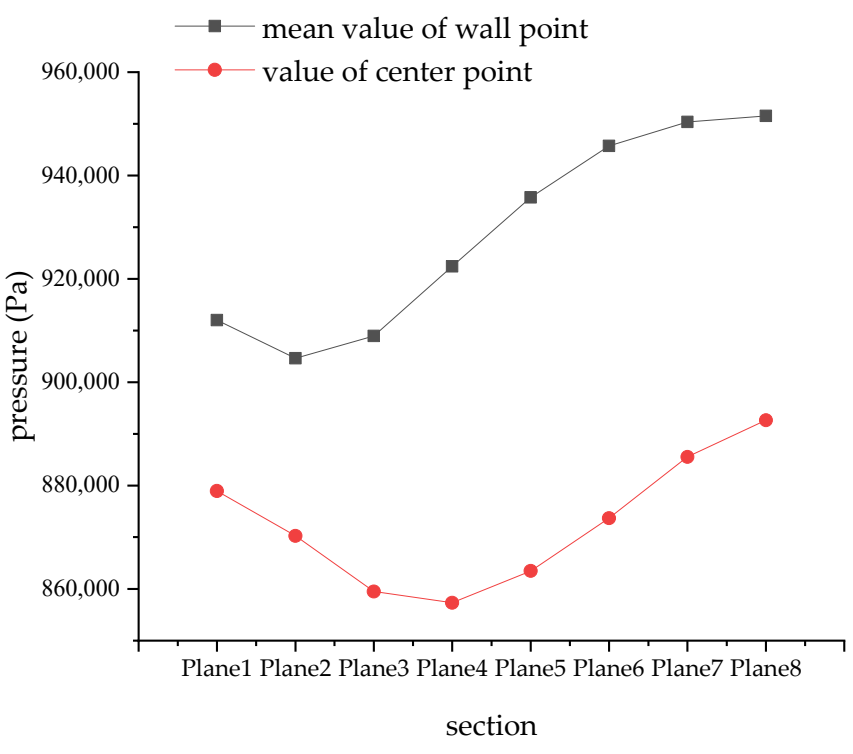

(a) Without cavitation

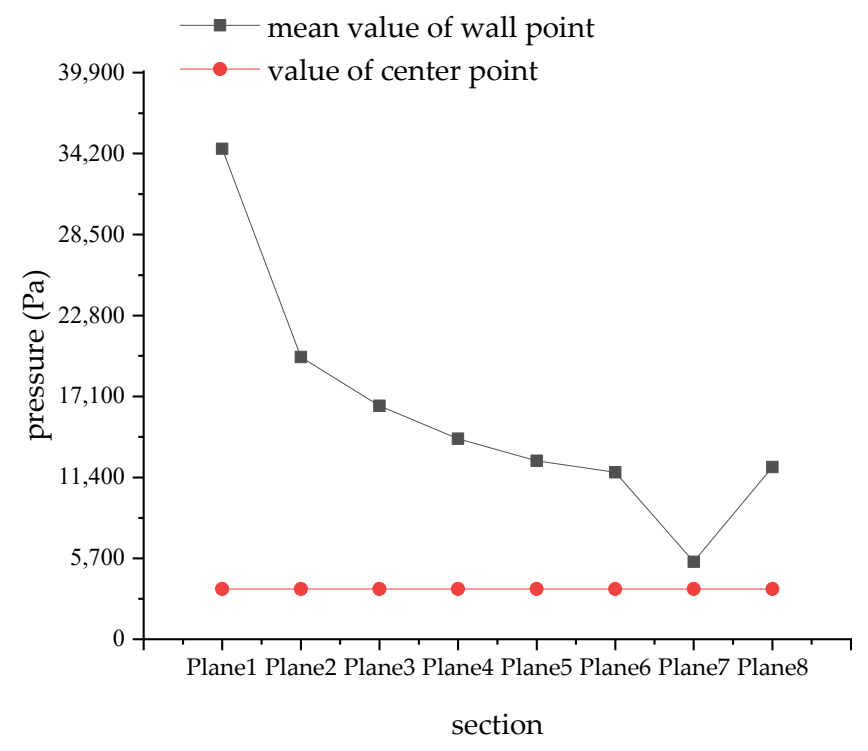

(b) Severe cavitation

Figure 6. Comparison of the pressure values of the different sections.

\section{Analysis of Transient Condition Results}

During the operation of the unit, when the load does not change, the main parameters of the unit, such as the water head, flow, speed, torque, shaft power, pressure, guide vane opening, etc., do not change, which is a stable working condition. When the hydraulic unit suddenly rejects the load, the flow rate will decrease sharply, the unit speed will rise rapidly from the rated speed, and then decrease after reaching the extreme value. After several pulsations, the unit will be stable in no-load operation, which is a load rejection process. During the load rejection process, the minimum pressure in the draft tube inlet is most likely to occur in the speed maximum moment, in which the cavitation is easy to present. Thus, three-dimensional CFD simulation at this moment was also conducted to analyze the pressure pulse inside the draft tube based on the calculation of the one-dimensional transient process.

In order to verify the accuracy of the calculation method, the $100 \%$ load rejection condition, testing the downstream water level, was calculated and analyzed. The working head is $296.47 \mathrm{~m}$, and the guide vane is closed normally.

In the one-dimensional calculation, the influence of the spiral casing and draft tube were considered. The spiral casing and draft tube were treated as equivalent. The equivalent volute diameter is the inlet diameter, which is $2.8 \mathrm{~m}$. The equivalent length is the distance from inlet to tongue, which is $24 \mathrm{~m}$. Draft tube equivalent is divided into three parts: straight cone section, elbow section and diffusion section. The diameter and length of the straight cone part are $2.7 \mathrm{~m}$, as well as the height of the straight cone section. The diameter of the bent shaft section is the average value of the equivalent diameter of the inlet and outlet section, which is $3.116 \mathrm{~m}$, and the length is the length of the elbow section, which is $6.354 \mathrm{~m}$. The diameter of the diffusion section is the average value of the equivalent diameter of inlet and outlet section of $4.645 \mathrm{~m}$, and the length is the length of the diffusion section.

When the unit loses the load, the guide vane starts to close after $10 \mathrm{~s}$ delay. The unit speed begins to rise after load rejection. The speed reaches the maximum value in $7.65 \mathrm{~s}$, and the maximum relative value of the speed is 1.407 times that of the rated speed. The speed begins to decline after the guide vane closes, as shown in Figures 7 and 8. In Figure 7, the relative opening degree means the ratio of the actual opening of the guide vane to the maximum opening. The outlet pressure of the ball valve is the first to increase in the whole process and reaches its maximum value of $4.37 \times 10^{6} \mathrm{~Pa}$ in $8.224 \mathrm{~s}$, and then decreases, as shown in Figure 9. The inlet pressure of the draft tube first decreases, and then increases, as 
shown in Figure 10. From the comparison between the calculation results and the test data, the calculation results are consistent with the test data in the transient process. The calculated error of the maximum speed is $1.4 \%$, and the pressure error at the outlet of the ball valve is $7.17 \%$. On the whole, the one-dimensional calculation results are reliable and effective.

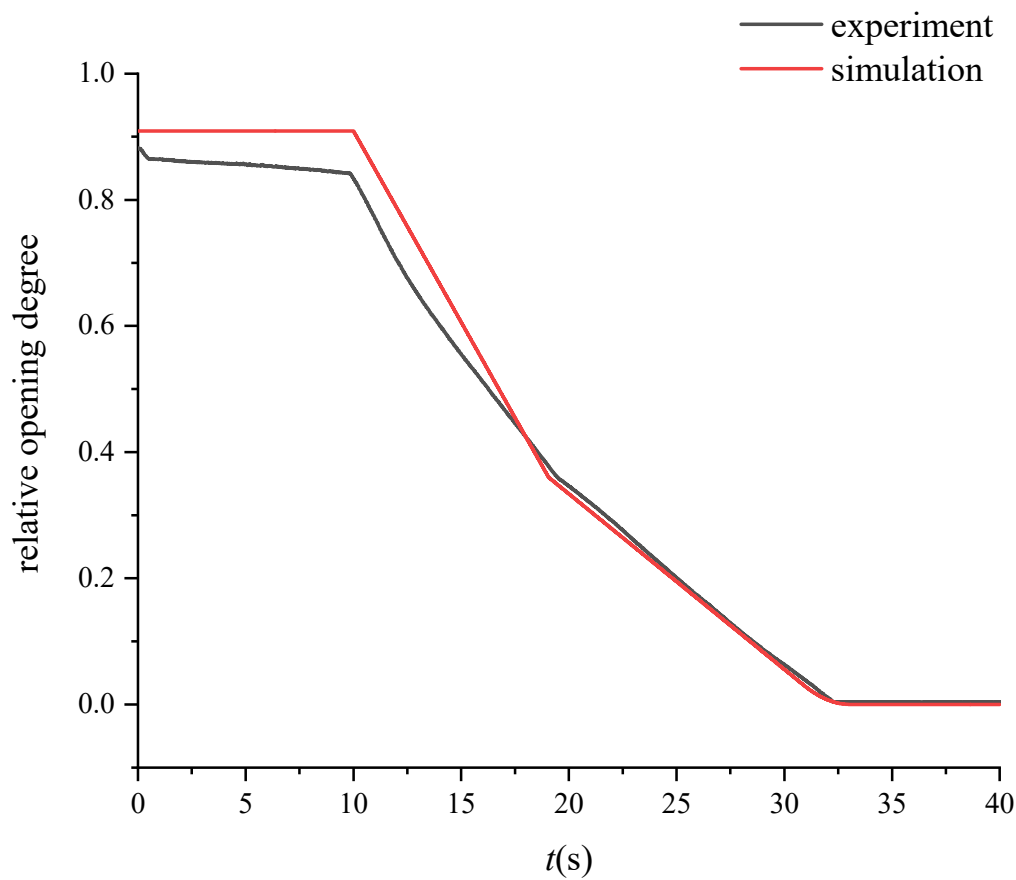

Figure 7. Comparison of the guide vane closing law.

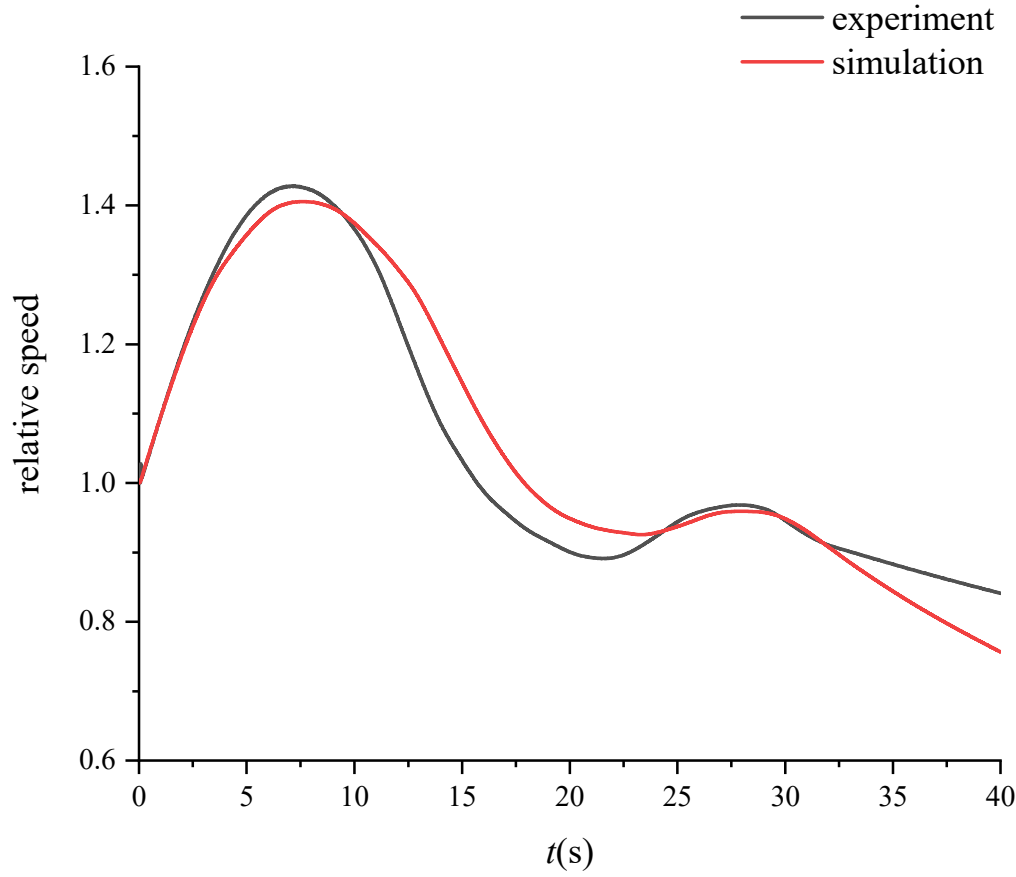

Figure 8. Comparison of the rotating speed. 


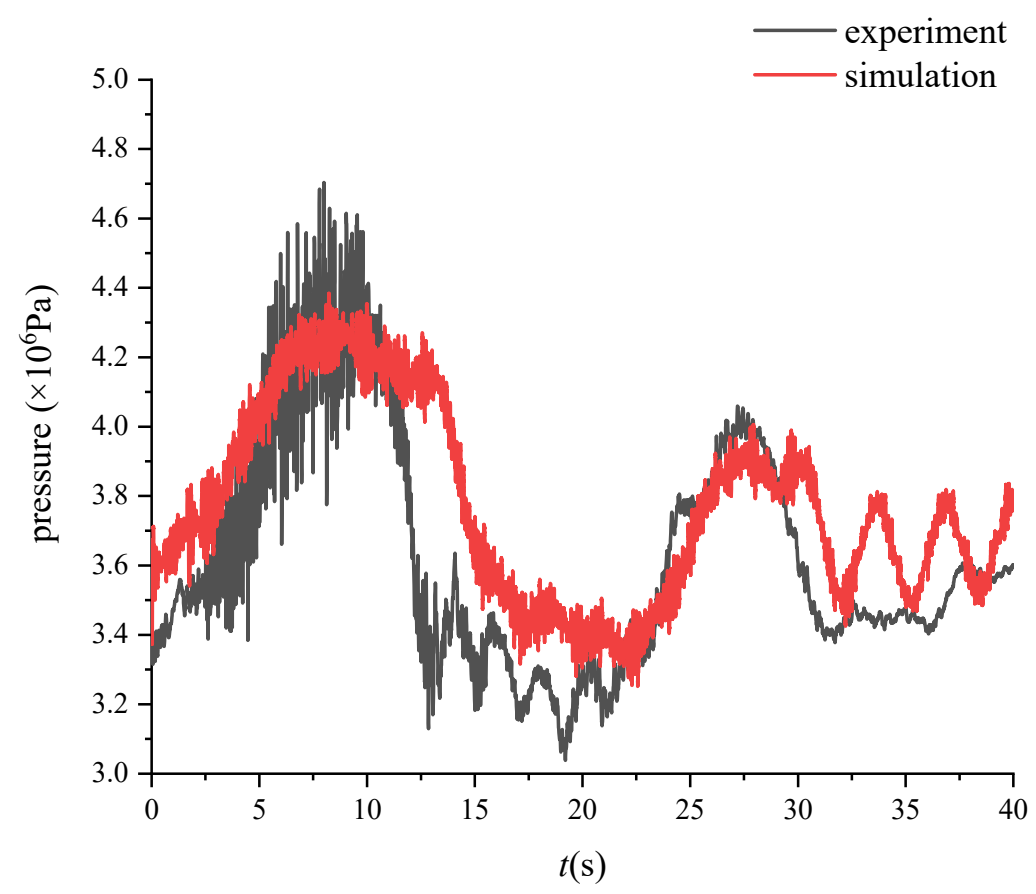

Figure 9. Comparison of the outlet pressure of the ball valve.

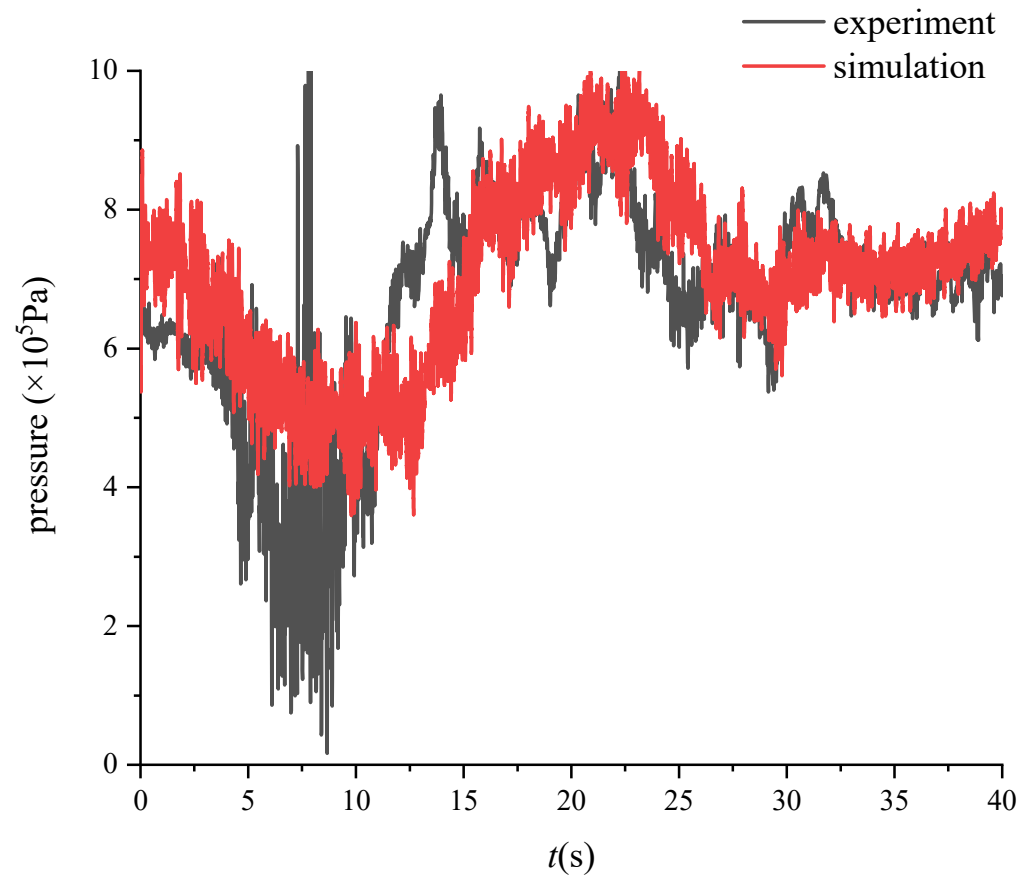

Figure 10. Comparison of the draft tube inlet pressure results.

According to the results of the one-dimensional calculation, in the process of load rejection, the lowest pressure of the draft tube inlet appears at the maximum speed, so the instantaneous value at this moment is taken to analyze the flow in the draft tube; that is, the three-dimensional simulation boundary conditions at the spiral casing inlet and draft tube outlet are set according to the calculated one-dimensional result at $6.9 \mathrm{~s}$ in the process of load rejection.

Due to the minimum pressure at the draft tube inlet being lower than the saturation pressure in the one-dimensional calculation (see Figure 10), the cavitation must be present in the draft tube during the load rejection, which was verified by the three-dimensional 
simulation (see Figure 11). The cavitation volume is represented by the iso-surface of the vapor volume fraction of 0.1 , which has been proven to have the most similar shape with the reality vortex rope [22]. A long vortex rope presents in the draft tube cone at time $t=6.9 \mathrm{~s}$; the cavitation is serious in the draft tube at this time, and the cavitation mainly occurs at the center of the draft tube.

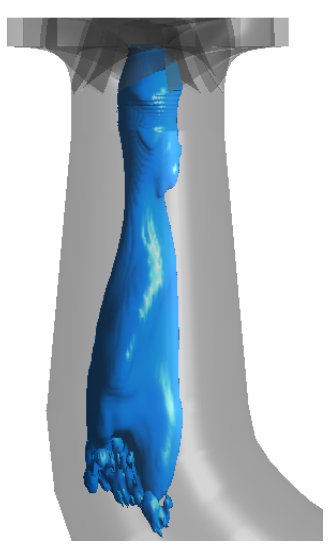

Figure 11. The cavitation area in the draft tube.

The pressure distributions at different sections in the draft tube at this dangerous moment are also shown in Figure 12. It shows that the pressure at each section center is lower than the saturated vapor pressure of $3540 \mathrm{~Pa}$, which means that cavitation appears. However, the pressure on the side wall is much larger than the saturated vapor pressure.

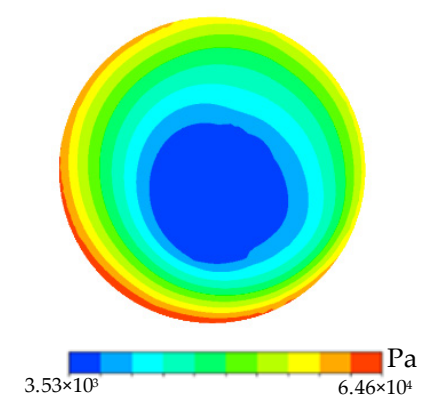

(a) Plane 1

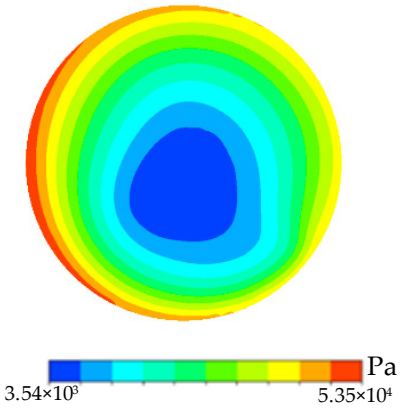

(c) Plane 3

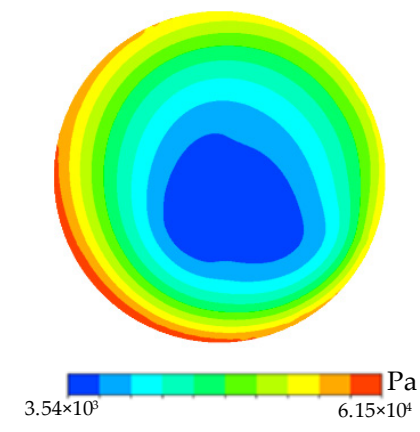

(b) Plane 2

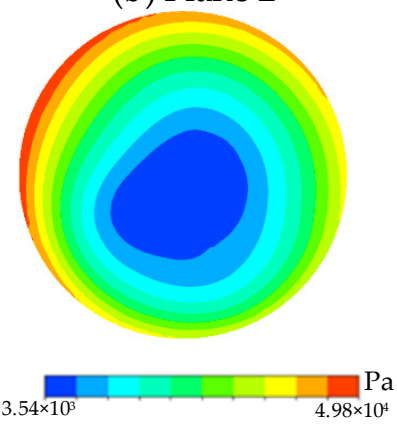

(d) Plane 4

Figure 12. Pressure distribution of the draft tube at $t=6.9 \mathrm{~s}$ on different sections.

Figure 13 presents the mean pressure of the wall point and the center point at each section at $6.9 \mathrm{~s}$ of transient process. The minimum pressure values at the center point of Planes 1-5 are $3540 \mathrm{~Pa}$, while the minimum pressure values of Planes 6-8 are greater than $3540 \mathrm{~Pa}$, indicating that the vortex rope is located at Planes $1-5$. It can be concluded that when there 
is a vortex rope passing through the section, the average pressure on the draft tube wall is quite different, with an average value of $2.8 \times 10^{4} \mathrm{~Pa}$ to $5 \times 10^{4} \mathrm{~Pa}$. At the Plane 6 section, the central point pressure is at the critical value of saturated vapor pressure, which is $3540.681 \mathrm{~Pa}$, which means the vortex rope has just disappeared. Thus, the average wall pressure at this section can be seen as the critical value of cavitation. That is to say, the maximum average pressure near the wall of the section to judge cavitation is about $5.1 \times 10^{4} \mathrm{~Pa}$. Based on the analysis in this paper, it can be seen that the critical average value of the wall monitoring pressure is about $5.25 \times 10^{4} \pm 1000 \mathrm{~Pa}$ under this condition. When the actual measured wall pressure is lower than the critical value, the occurrence of cavitation can be preliminarily judged. Further confirmation can be carried out by CFD simulation.

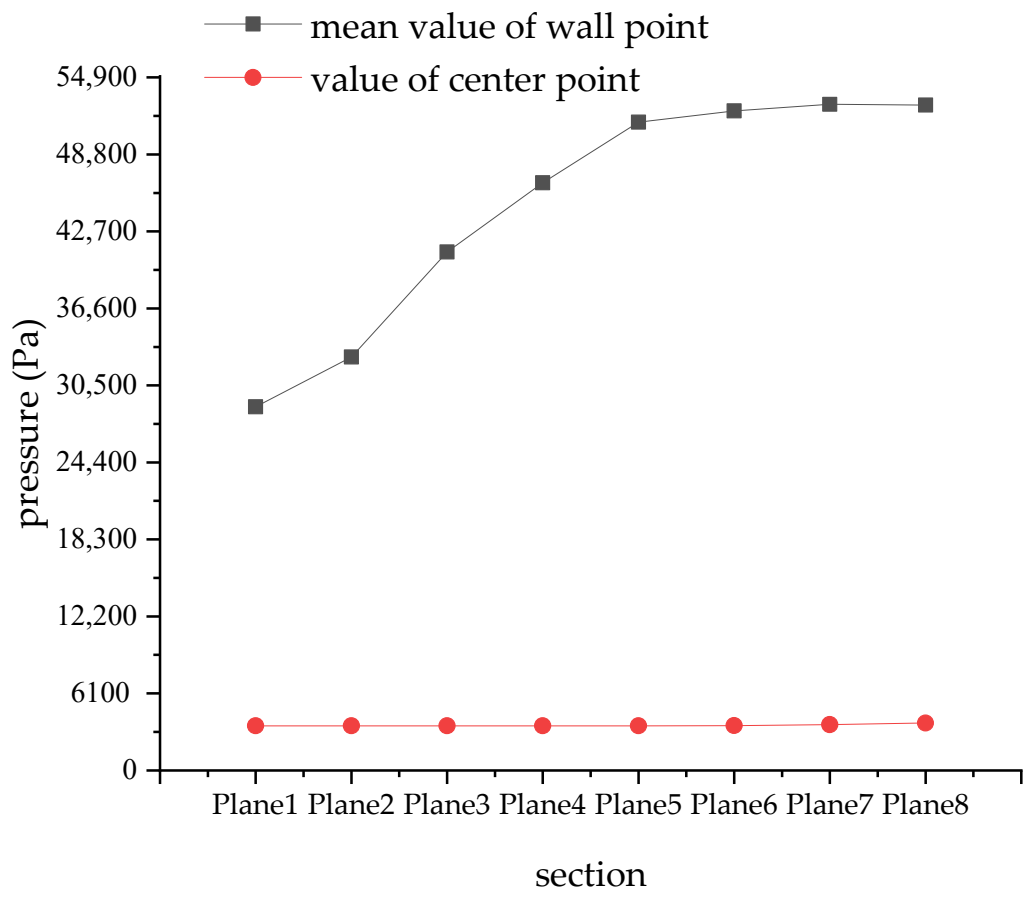

Figure 13. Pressure values of the different measuring points at $t=6.9 \mathrm{~s}$.

\section{Conclusions}

In this paper, the pressure in the draft tube of a pump-turbine are studied and analyzed under the steady-state condition and load rejection transient process.

The average wall pressure calculated and monitored in the in the steady-state condition was first carried out; the results showed the central point pressure being obviously different from that near the wall, and that the center point is the most accurate point to judge cavitation.

Under the transient condition, the moment of cavitation occurrence is identified firstly (for example, the load rejection condition studied in this paper is the point of the maximum speed), and then the instantaneous value of the pressure at that time is calculated and compared. The average pressure at the monitored points in $6.9 \mathrm{~s}$ under the transient condition was obtained. Combined with the "critical values" of cavitation present at the central point, the mean wall pressure "critical value" was obtained. For this research, the "critical" wall pressure pulsation is $5.25 \times 10^{4} \pm 1000 \mathrm{~Pa}$.

For application in engineering, by comparing the average value of the wall pressure pulsation obtained from the transient or steady-state tests with the "critical" value proposed in this paper, the critical conditions of cavitation can be preliminarily predicted. The results of this paper provide a new method for cavitation identification based on the pressure value from the wall monitoring points in engineering. 
Author Contributions: Conceptualization, J.Y.; methodology, J.Y. and Y.L.; investigation, J.Y. and D.L.; writing—original draft preparation, J.Y.; writing-review and editing, Z.W.; supervision, Z.W.; project administration, D.L. and Y.L. All authors have read and agreed to the published version of the manuscript.

Funding: This work was supported by National Natural Science Foundation of China (No: 51876099).

Data Availability Statement: Not applicable.

Acknowledgments: Thanks for the test data provided by relevant units and the support of National Natural Science Foundation of China (no. 51876099).

Conflicts of Interest: The authors declare no conflict of interest.

\section{References}

1. Yang, J.; Hu, J.; Zeng, W. Transient pressure pulsations of prototype Francis pump-turbines. J. Hydraul. Eng. 2016, 47, 858-864.

2. Xia, L.; Cheng, Y.; You, J.; Zhang, X.; Qian, Z. Mechanism of the S-Shaped Characteristics and the Runaway Instability of Pump-Turbines. J. Fluids Eng. 2017, 139, 031101. [CrossRef]

3. Trivedi, C.; Cervantes, M.; Gandhi, B.; Dahlhaug, O. Transient pressure measurements on a high head model Francis turbine during emergency shutdown, total load rejection, and runaway. J. Fluids Eng. 2014, 136, 121107. [CrossRef]

4. Luo, X.; Li, W.; Feng, J. Simulation of runaway transient characteristics of tubular turbine based on CFX secondary development. Trans. Chin. Soc. Agric. Eng. 2017, 33, 97-103.

5. Liu, J.; Liu, S.; Sun, Y.; Wu, Y.; Wang, L. Three dimensional flow simulation of load rejection of a prototype pump-turbine. Eng. Comput. 2013, 29, 417-426.

6. Wang, Z.; Zhou, L.; He, C. Pressure oscillations in a hydraulic turbine draft tube. J. Tsinghua Univ. (Sci. Technol.) 2006, 45, $1138-1141$

7. Tanaka, T.; Tsukamoto, H. Transient Behavior of a Cavitating Centrifugal Pump at Rapid Change in Operating Conditions-Part 1: Transient Phenomena at Opening/Closure of Discharge Valve. J. Fluids Eng. 1999, 121, 841-849. [CrossRef]

8. Ruprecht, A.; Helmrich, T. Simulation of the Water Hammer in a Hydro Power Plant Caused by Draft Tube Surge. In Proceedings of the ASME/JSME 2003, Joint Fluids Summer Engineering Conference, Honolulu, HI, USA, 6-10 July 2003; pp. $2811-2816$.

9. You, J.; Lai, X.; Zhou, W.; Cheng, Y. 3D CFD simulation of the Runaway process of a Pelton turbine. J. Power Energy 2016, 230, 234-244. [CrossRef]

10. Li, Z.; Bi, H.; Karney, B.; Wang, Z.; Yao, Z. Three dimensional transient simulation of a prototype pump-turbine during normal turbine shutdown. J. Hydraul. Res. 2017, 55, 1-18. [CrossRef]

11. Zhang, X.; Cheng, Y.; Yang, J.; Xia, L.; Xu, L. Simulation of the load rejection transient process of a Francis turbine by using a 1-D-3-D coupling approach. J. Hydrodyn. Ser. B 2014, 26, 715-724. [CrossRef]

12. Cherny, S.; Chirkov, D.; Bannikov, D.; Lapin, V.; Skorospelov, V.; Eshkunova, I.; Avdushenko, A. 3D numerical simulation of transient processes in hydraulic turbines. In Proceedings of the IOP Conference Series-Earth and Environmental Science, Timişoara, Romania, 20-24 September 2010; Volume 12.

13. Yang, J.; Hu, Q.; Wang, Z.; Ding, J.; Jiang, X. Effects of inlet cavitation on swirling flow in draft-tube cone. Eng. Comput. 2018, 35, 1694-1705. [CrossRef]

14. Nicolet, C. Hydroacoustic Modelling and Numerical Simulation of Unsteady Operation of Hydroelectric Systems. Ph.D. Thesis, École Polytechnique Fédérale de Lausanne, Swiss, Lausanne, Switzerland, 2007.

15. Nicolet, C.; Arpe, J.; Avellan, F. Identification and modeling of pressure pulsations of a Francis turbine scale model at part load operation. In Proceedings of the 22nd IAHR Symposium on Hydraulic Machinery and Systems, Stockholm, Sweden, 29 June-2 July 2004.

16. Alligné, S. Forced and Self Oscillations of Hydraulic Systems Induced by Cavitation Vortex Rope of Francis Turbines. Front. Artif. Intell. Appl. 2011, 255, 65-73.

17. Meng, L. The Study of Cavitation Influence on Centrifugal Pump Startup Process. Ph.D. Thesis, China Agricultural University, Beijing, China, 2016.

18. Zhang, X.; Cheng, Y.; Xia, L.; Yang, J. CFD simulation of reverse water-hammer induced by collapse of draft-tube cavity in a model pump-turbine during runaway process. IOP Conf. 2016, 49, 052017. [CrossRef]

19. Pejovic, S.; Zhang, Q.; Karney, B.; Gajic, A. Analysis of Pump-Turbine “S” Instability and Reverse Waterhammer Inci-dents in Hydropower Systems. In Proceedings of the 4th International Meeting on Cavitation and Dynamic Problems in Hydraulic Machinery and Systems, Belgrade, Serbia, 26-28 October 2011.

20. Luo, X.; Ji, B.; Tsujimoto, Y. A review of cavitation in hydraulic machinery. J. Hydrodyn. 2016, 28, 335-358. [CrossRef]

21. Zwart, P.J.; Gerber, A.G.; Belamri, T. A two-phase flow model for predicting cavitation dynamics. In Proceedings of the 5th International Conference on Multiphase Flow, Yokohama, Japan, 30 May-4 June 2004.

22. Dular, M.; Bachert, R.; Stoffel, B.; Širok, B. Experimental evaluation of numerical simulation of cavitating flow around hydrofoil. Eur. J. Mech. B/Fluids 2005, 24, 522-538. [CrossRef] 